Fuel cells can power zero emission cars

and supercapacitors help to reduce

obstacles for widespread adoption of the

technology

\section{Supercapacitors boost the fuel cell car}

\author{
Fritz Gassmann, Rüdiger Kötz and Alexander Wokaun \\ Paul Scherrer Institute, Villigen, Switzerland
}

$N^{\circ}$ o matter where you live it cannot have escaped your attention that our planet is struggling. There may be some who are sceptical but most of us can have little doubt that if we do not mend our ways, severe changes of global climate lie ahead. One of the most obvious major contributors to the deteriorating situation are the some 750 million registered vehicles worldwide that emit roughly 4 billion tons of carbon dioxide each year and contribute $15 \%$ to the anthropogenic emissions. In addition to their impact on the global climate, several other facts are motivating car manufacturers to investigate ways of reducing emissions drastically: declining oil reserves, their location in politically unstable regions, and health hazards posed by secondary emissions of nitrogen oxides, hydrocarbons, and particulates. Today's most promising solution for these problems would be cars powered by fuel cells with solar hydrogen as the ultimate energy carrier (cf preceeding article). However, a number of obstacles are delaying widespread adoption of this technology including high costs, the weight and volume of today's fuel cells, security concerns related to hydrogen storage tanks, and the missing infrastructure needed for the production and distribution of hydrogen.

All these engineering and economic problems could be minimised if cars could be built without having excessive power, e.g. a 1 ton compact-class car with $20 \mathrm{~kW}$ continuous mechanical power at the wheel. Imagine such a car that could reach the maximum allowed speeds in most countries $(120 \mathrm{~km} / \mathrm{h}$ or $75 \mathrm{mph})$,

\section{Box 1}

\section{Physical relations for capacitors}

$\mathrm{U}_{1}$

Both electrodes have a surface $A$ (in $\mathrm{m}^{2}$ ) separated by distance $\mathrm{d}$ (in $\mathrm{m}$ ). The applied voltage $\Delta \mathrm{U}$ (in Volt) creates an electric field $\mathrm{E}=\Delta \mathrm{U} / \mathrm{d}$ storing the electrical energy. Capacitance $C$ in Farad (F) and stored energy J in Ws is:

$$
C=\varepsilon_{0} \cdot \varepsilon_{\mathrm{r}} \frac{\mathrm{A}}{\mathrm{d}} \quad \mathrm{J}=\frac{1}{2} \mathrm{C} \cdot \Delta \mathrm{U}^{2}
$$

where $\varepsilon_{\mathrm{r}}$ (e.g. 1 for vacuum or 81 for water) is the relative dielectric constant which depends on the material placed between the two electrodes and $\varepsilon_{0}=8.85 \cdot 10^{-12} \mathrm{~F} / \mathrm{m}$ is a fundamental constant. climb over every pass (it would climb $6 \%$ at a speed of $80 \mathrm{~km} / \mathrm{h}$ or $50 \mathrm{mph}$ ) and transport people to their working places or shopping centres in the accustomed time (remember that powerful engines can not help to lubricate a traffic jam). However, the hypothetical $20 \mathrm{~kW}$ car would need around 30 seconds to accelerate to $100 \mathrm{~km} / \mathrm{h}(62 \mathrm{mph})$ and would, therefore, only be accepted by a few pioneers. The common technical answer of car manufacturers to this rather irrational but common human desire for power is oversizing engine power by a factor of about two to eight. The price customers pay for this solution is twofold: Powerful engines are more expensive, and, since these engines work below their optimum efficiency most of the time, their fuel consumption is high, causing increased operating costs and at the same time increased environmental damage.

\section{Power reserve for 15 seconds}

How do we escape from this trap? One solution is supercapacitors (also called supercaps, ultracapacitors or electrochemical double layer capacitors, EDLC) which offer the possibility for electric cars to reconcile the widespread wish for power with environmental concerns [1]. Instead of designing the primary power system to deliver a maximum of e.g. 73 $\mathrm{kW}$ (100 horsepower), it could be designed for an average power of only $20 \mathrm{~kW}$, with supercapacitors acting as power reserves to deliver peak power during a limited time. With $53 \mathrm{~kW}$ of additional power delivered over $15 \mathrm{sec}$ onds, most power display behaviour patterns could be satisfied: The car would accelerate to $100 \mathrm{~km} / \mathrm{h}(62 \mathrm{mph})$ in less than 15 seconds, passing other cars over short distances would be possible, and enough rubber could be burned with starts to satisfy the speedster. Additional peak power of 53

$\mathrm{kW}$ over 15 seconds means access to an energy buffer of $220 \mathrm{Wh}$, an amount of energy costing a few cents when taken from the electric power grid.

Could the lead-acid batteries normally employed for the electric starter motors be used for this purpose? Two main limitations must be considered. Though a normal $12 \mathrm{~V}$ battery with a capacity of $60 \mathrm{Ah}$ stores enough energy $(720 \mathrm{Wh})$, the current should not exceed about $150 \mathrm{~A}$, limiting the power to $1.8 \mathrm{~kW}$, far below the desired $53 \mathrm{~kW}$. Secondly, chemical reactions are taking place whenever current flows into or out of a battery. In principle the transformation from bulk lead sulfate into dissolved sulfate ions is completely reversible, but the decreasing mechanical stability of the lead sulfate electrode leads to degradation after several hundred charge/discharge cycles. In spite of considerable research on new battery concepts over the last decade, no battery has been found that would circumvent these limitations.

With capacitors used in practically all electronic devices, e.g. to smooth ripples on the DC current in power supply units, power and stability are no problem. As energy is stored by a purely physical process in electric fields without involving chemical transformations, the number of loading cycles does not limit their lifetime. Furthermore, charge- or discharge-currents as measured in relation to the energy content, are about four decades higher 
than with batteries. The big disadvantage of traditional capacitors is their relatively small capacitance. This characteristic value (measured in Farads) relates to their energy content when multiplied by half the square of the applied voltage (see Box Physical relations for capacitors). A typical large electrolyte-capacitor used for power supplies has a capacitance of the order of $20^{\prime} 000$ microFarad and allows a maximum voltage of about $20 \mathrm{~V}$, storing only 4 Joules of energy, equivalent to $4 \mathrm{Ws}$ or $0.0011 \mathrm{Wh}$. With a mass of about $20 \mathrm{~g}$ for such an element, roughly 4 tons would be needed to store the required energy of $220 \mathrm{Wh}$ ! For an overview of frequently used capacitors in electronic devices see Fig. 1.

\section{Supercapacitors combine advantages of battery and capacitor}

Over about the last decade, double-layer capacitors with unprecedented large capacitance have been developed in various research laboratories, and several companies have already started commercial production. At the Paul Scherrer Institute in Villigen, Switzerland, Rüdiger Kötz and his group have developed an electrode in collaboration with the Swiss company montena (recently merged with Maxwell). Towards the end of 2001, one of their supercapacitors reached a capacitance of 1600 Farad at a maximum voltage of $2.5 \mathrm{~V}$. They store 5000 Joules or $1.4 \mathrm{Wh}$ within a volume of roughly $0.3 \mathrm{~L}$ ( $5 \mathrm{~cm}$ diameter, $14 \mathrm{~cm}$ long) weighing $320 \mathrm{~g}$. To store the required $220 \mathrm{Wh}, 160$ supercapacitors weighing $50 \mathrm{~kg}$ would suffice. With their small internal series resistance of $0.0014 \mathrm{Ohm}$, they can produce or absorb peak currents of over 300 A. Due to the excessively high currents at low voltages, operation is normally restricted to between $50 \%$ and $100 \%$ of maximum voltage and therefore, only $75 \%$ of the energy capacity is used (due to the quadratic relationship between voltage and energy content, the residual energy equates to only $25 \%$ at $50 \%$ of the voltage). This restriction and also losses in power converters result in increasing the number needed from 160 to about 250 supercapacitors, weighing nearly $100 \mathrm{~kg}$, including cables and electronics.

\section{How to build a large capacitor?}

In a capacitor, energy is stored within the electric field between its electrodes in the following way: The application of a voltage between the electrodes results in the flow of electrons towards the negative electrode, and away from the positive electrode (see Box Physical relations for capacitors). This transport of electric charge towards and from the respective electrodes is equivalent to an electric current. Multiplication of this current by the applied voltage gives the power flowing into the electric field between the electrodes. The capacitance is determined by the geometric dimensions of the device and by the relative dielectric constant of the applied isolator foil. While this latter value lies between 1 for air to 81 for water and up to several 1000 for ceramics, more can be achieved by manipulating the geometry. Capacitance is proportional to the surface area of the electrodes divided by their separation distance, giving units of length (at the beginning of radio telecommunication, capacitors were therefore measured in $\mathrm{cm}$ ). Simple capacitors consisting of two parallel plates reach only very small capacitances, of the order of pico-Farad $\left(1 \mathrm{pF}=10^{-12} \mathrm{~F}\right)$, and are used in high frequency technology. Even when loaded to $1000 \mathrm{~V}$, the energy content of such plate capacitors is only of the order of micro-Ws (see lowest picture in Fig. 1).

To increase the geometrical part of the capacitance, the surface area of the electrodes can be increased by rolling long stripes of conducting material, and at the same time, the isolating layer of material with high dielectric constant in between them is manufactured as thin as possible. With this technology, rolled capacitors of the order of 0.1 micro-Farad $\left(1 \mu \mathrm{F}=10^{-6} \mathrm{~F}\right)$ resisting voltages up to about $1000 \mathrm{~V}$ can be produced, storing around $0.05 \mathrm{Ws}$ of energy (see second lowest picture in Fig. 1).

$\nabla$ Fig. 1: Capacitors covering a range of 15 decades.

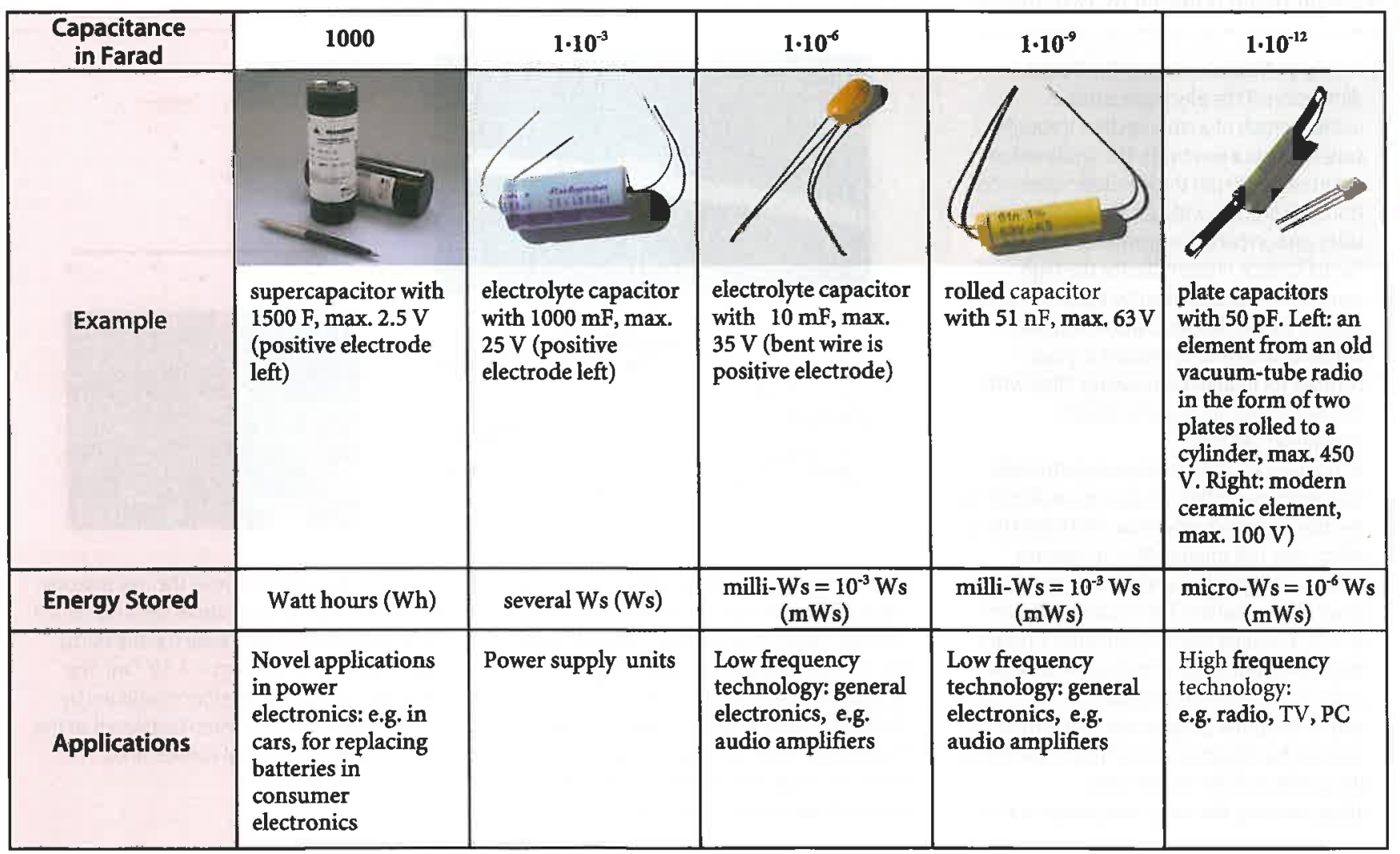


Another possible way to increase the geometrical part of the capacitance is to replace one of the electrodes by a liquid electrolyte (an electrically conducting gel) in order to achieve immediate geometrical contact on the atomic scale to the surface of a metallic electrode. A thin oxidic layer on the surface of the metal electrode serves as an isolator and separates the electrodes to distances of the order of nanometres, pushing capacitance into the tens of milli-Farad range $\left(1 \mathrm{mF}=10^{-3} \mathrm{~F}\right)$ at maximum voltages of around $20-40 \mathrm{~V}$. These so called electrolyte capacitors can store up to several Ws of energy (see middle and second highest picture in Fig. 1).

Increasing the surface area by rolling and minimizing the separation distance to the molecular range long seemed the ultimate limit in the production of large capacitors. However, fractal geometry has opened amazing, and counterintuitive, new possibilities of how to scramble e.g. the surface of a footballfield into a $1 \mathrm{~mm}$ thick layer above a sheet of paper the size of the journal you are reading right now. By increasing the electrode surface about $100^{\prime} 000$-fold in this way, electrolyte capacitors with thousands of Farad can be built.

\section{The mystery of the inner surface}

Back at the beginning of the 20th century, "mathematical monsters" were invented: by an iterative process involving ever smaller lengths, linear objects with infinite length were created which could be fitted into a limited area, that was not at all filled up by the object. One of the most famous of these objects is the curve invented by the Swedish mathematician N.F.H von Koch in 1906: A line of unit length is divided into three equal parts, the middle segment being replaced by two lines of length one third each.
Obviously, repeated iteration leads to an ever longer path, because its length is multiplied by $4 / 3$ with every iteration. On the other hand, the resulting line never exceeds a square of unit side length, nor does it need a considerable fraction of the square, because even a very long mathematical line still has zero surface. However, more and more points on the line are located close to an

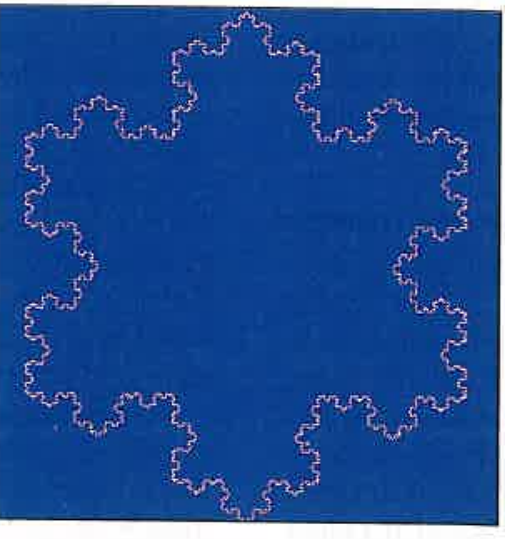

A Image: The Kock island. increasing number of points belonging to the surface of the square, yet eyeball inspection gives the impression that the geometrical object somehow combines the characteristics of a line and of a surface. With fractal dimension $\mathrm{D}$, this qualitative property is put into an objective mathematical framework (see Box: Fractal Dimension)

Applying fractal geometry to increase the capacity [4], the surface of the electrodes has been roughened by using soot particles in contact with the flat metallic carrier-foil of the electrode (see Fig. 2a: micrograph of cross section). A measurement of the fractal dimension of the borderline in a cross-section perpendicular to the electrode yields $\mathrm{D} \approx 1.6$ (see Fig. $2 b, c, d)$. Using a general topological formula yields $D_{S} \approx 2.6$ for the overall dimension of the electrode surface. As self similarity of every physical object is limited to a maximum range between its macroscopic and the atomic scale, the concept of fractal dimension is also restricted to a limited range of length scales. Considering $D_{S} \approx 2.6$ as representative between the macroscopic scale of the electrode (of the order of $0.1 \mathrm{~m}$ ) and the microscopic scale (in the order of $1 \mathrm{~nm}$ ), self similarity can be assumed to hold over 8 decades. The electrode surface is therefore multiplied by 10 to the power of $8^{*}(2.6-2)$ or $60^{\prime} 000$. In combination with the extremely thin electro-chemical double layer (around $1 \mathrm{~nm}$ ), unprecedented capacitances can be reached.
Fig. 2 Measurement of the fractal dimension of the electrode surface. a) Micrograph of a cross section through a supercapacitor electrode. The white stripe is a part of the $30 \mu \mathrm{m}$ thick metallic carrier-foil (total foil is $0.1 \mathrm{~m}$ wide, $2 \mathrm{~m}$ long). On both sides carbon particles provide a complex fractal surface responsible for the high capacity. The space taken by the green resin used to fix the delicate carbon structure before cutting and to provide a good contrast for imaging is normally filled with the electrolyte (an organic solvent containing salt ions).

b) Borderline of the cross section through the electrode surface in (a) to be analyzed by the box-counting procedure, illustrated for a tiling with 128 squares: $M=56$ squares (filled with light blue colour) are necessary to cover the borderline. Their side lengths are $N=11.3$ (square root of 128) times smaller than the length scale of the whole picture. c) The box-counting procedure is repeated with a computer program for different $N$. The average fractal dimension of the borderline is the gradient of the straight line

approximating the measured points in this a)

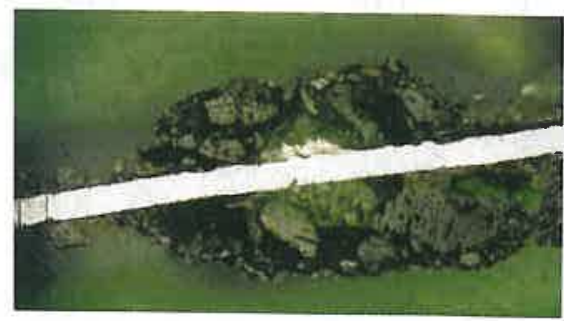

b)

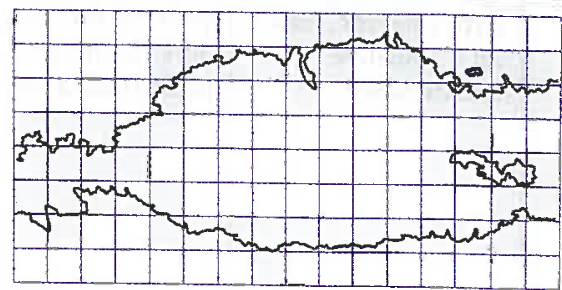

c)

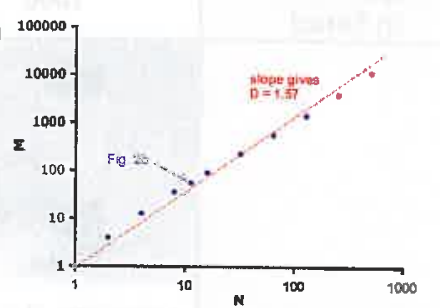

d)

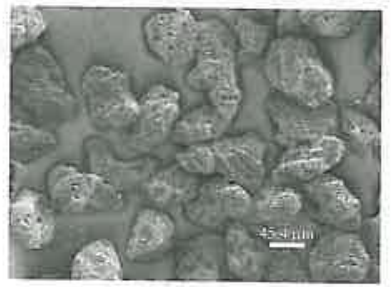

$\log (M)$ over $\log (N)$ plot, giving $D \approx 1.6$. This same dimension was measured in the lengthinterval covering nearly 3 decades between $0.6 \mathrm{~mm}$ (length of micrograph in Figs $2 a, b$ ) and about $1 \mu \mathrm{m}$ (fine structure in Fig. $2 \mathrm{~d}$ ). d) Carbon particles as seen with an electron microscope show roughness also in the $1 \mu \mathrm{m}$ scale. It is assumed that the above indicated fractal dimension $D$ holds over the entire range of 8 decades between the macroscopic scale (i.e. the geometric size of the order of 0.1 $\mathrm{m})$ and the microscopic scale (i.e. the micropores in the order of $\left.1 \mathrm{~nm}=1 \cdot 10^{-9} \mathrm{~m}\right)$. The electrode surface is therefore multiplied by $10^{8^{*} 0.6}$ or about $60^{\prime} 000$ when compared to the normal two-dimensional surface of $0.2 \mathrm{~m}^{2}$. 


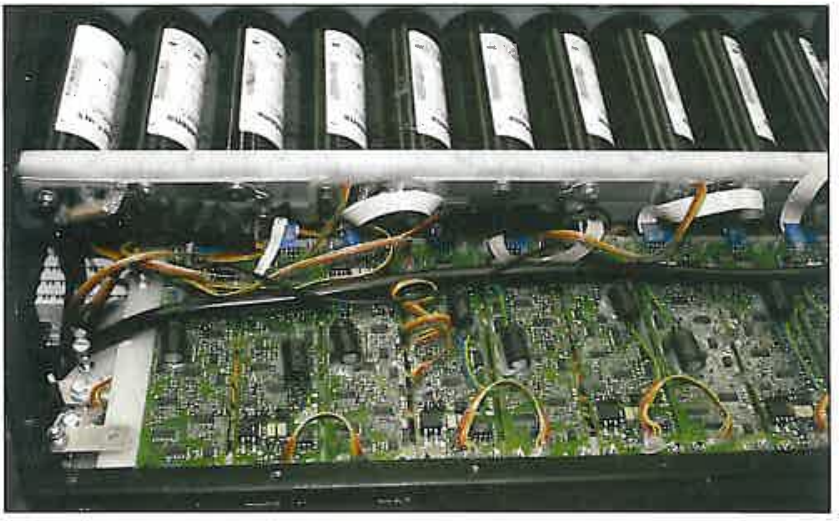

A Fig. 3: Module with 142 supercapacitors and electronics for voltage balancing that was placed under the hood of the Hy.Power. A similar module with 140 supercapacitors was placed below the rear seats.

\section{Power box for an electric car}

To demonstrate the practicability of using supercapacitors to power a car, two modules were assembled with 140 and 142 supercapacitors (Fig. 3 shows one of them) developed in the framework of a collaboration between the Paul Scherrer Institute and the company montena SA in Rossens, Switzerland. This capacitor bank is capable of delivering $50 \mathrm{~kW}$ over 15 seconds. We arranged the 282 supercapacitors in pairs of elements connected in parallel. These pairs were then connected in series, leading to a voltage range between about 175 and $350 \mathrm{~V}$, with each element operating between 1.25 and $2.5 \mathrm{~V}$. When starting with fully loaded supercapacitors, the current is $150 \mathrm{~A}$, supplying a power of $50 \mathrm{~kW}$, and this increases to $300 \mathrm{~A}$ at the lower bound of operation voltage. As current is distributed between two supercapacitors connected in parallel, only half of the current flows through each element, far below the maximum ratings. The voltage range $175-350 \mathrm{~V}$ for this arrangement is favourable for further processing in a DC/DC power converter, developed by the Swiss Federal Institute of Technology (ETH) in Zürich, that transforms the voltage to a stabilized value. However, the series connection of a large number of capacitor pairs resulted in a problem: Small differences in the characteristics of the individual elements lead to different self-discharge rates (without involving external current), resulting in

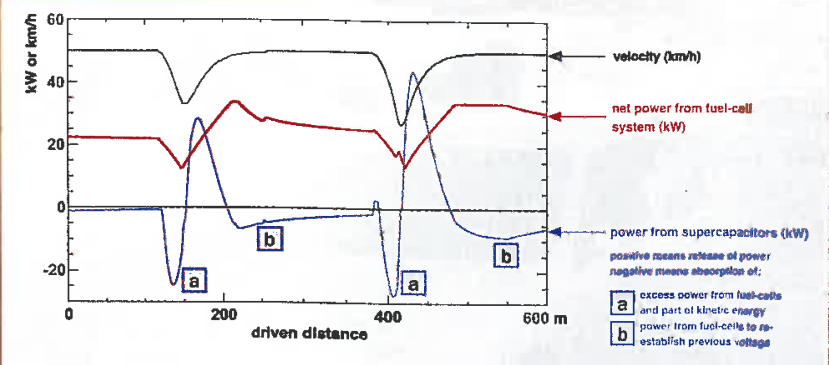

- Fig. 4: Simulation of power flows for an uphill drive on a steep mountain road through a typical double-curve. In both curves, velocity has to be reduced from 50 to about $30 \mathrm{~km} / \mathrm{h}$ (black curve). The net power of the fuel cell system is regulated with maximum gradients of $3 \mathrm{~kW} / \mathrm{s}$ (red curve). Excess power of the fuel cells and braking energy is stored in the supercapacitors (blue curve, large negative swings marked (a)). Accelerating after the curves is based mainly on supercapacitor power (large positive swings). After the acceleration phases, the supercapacitor system is refilled with energy from the fuel cells (smaller negative swings marked (b)).

steadily growing asymmetries between the voltages across different pairs of supercapacitors. As the maximum voltage of $2.5 \mathrm{~V}$ must not be exceeded, charge asymmetries have to be eliminated by redistribution between neighbouring pairs of capacitors. This task was accomplished by electronics specially developed for this purpose by ETH Lausanne, Switzerland.

The introduction of supercapacitors for use as a power buffer demands advanced power management that properly directs the energy flows. Instead of a direct coupling between the gas pedal and the fuel supply, an intelligent controller was developed by Paul Rodatz at ETH Zürich that translates a power demand (deduced from the position of the gas and brake pedals) into energy flows from the fuel cells, from the supercapacitors or from both systems into the driving engine. Conversely, during braking, power is taken from the engine, serving as an electric generator, and is fed into the supercapacitors. For various US and European mixed driving cycles, it has been shown that fuel consumption can be cut by $5-15 \%$ due to this regenerative mode of braking. The supercapacitor voltage is regulated based on a strategy adapted to the driving situation: At low speeds, the supercapacitors should be

\section{Box 2}

\section{Fractal Dimension}

Assume a first measurement process performed with a stick of unit length. Obviously, it can be placed exactly once over the curve, and its finer structures are not detected. By repeating the measuring process with a measuring stick three times shorter than before $(N=3)$, it can be placed four times $(M=4)$ onto the "Koch curve". The same reasoning holds for any successive refinement of the measurement process. Consider now the well known definition $M=N^{D}$, holding for all smooth geometrical objects, giving $\mathrm{D}=0$ (point), $\mathrm{D}=1$ (line), $\mathrm{D}=2$ (area) or $\mathrm{D}=3$ (cube) when measured with successively smaller length scales. Applying the same definition for the "Koch curve" with $\mathrm{N}=3$ and $M=4$ leads to a "fractal" dimension $D=(\log 4) /(\log 3) \approx 1.2618$. Over recent decades the concept of fractal dimension found numerous interesting applications in such diverse fields as geology, ecology, cosmology, physics and chemistry, medical sciences and economics $[2,3]$.

Step 0: Line of unit length is divided into 3 equal intervals.

Step 1: The central interval is replaced by two lines of length $1 / 3$, so increasing total length of the object by a factor of $4 / 3=1.3333$.

step 0:

$1 / 3 \quad 1 / 3 \quad 1 / 3$

For the next step, the procedure is repeated for all four intervals, and so on. With an increasing number of iterations $n$, the length of the line gets therefore $1.3333^{\mathbf{n}}$ and so exceeds every arbitrary large number, i.e. its length diverges towards infinity. A similar procedure leads to the well known Koch snowflake or Koch island (see picture). 


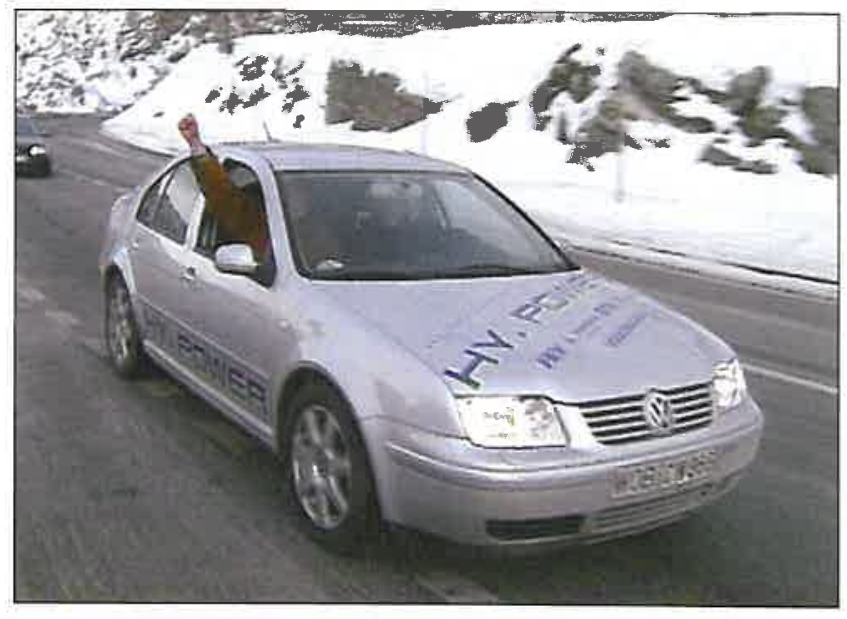

$\Delta$ Fig. 5: Hy. Power arriving on the $2005 \mathrm{~m}$ (6580 ft.) high Simplon pass in January 16, 2002 during harsh weather conditions.

nearly full to offer enough energy for acceleration, while near maximum speed, supercapacitor voltage should be low, providing enough capacity for recovery of kinetic energy during braking. As an additional facet, capacitors should be nearly full during uphilldriving independent of speed, to guarantee a power reserve for steeper sections of the road. Obviously, the supercapacitors should be loaded only when power reserves are available from the fuel cell system. A simulation of power management for driving along an uphill S-curve of a mountain road is shown in Fig. 4. Just before entering the The introduction of curves, velocity is reduced from $50 \mathrm{~km} / \mathrm{h}(30 \mathrm{mph})$ to supercapacitors for about $30 \mathrm{~km} / \mathrm{h}(20 \mathrm{mph})$. At use as a powel cell system is reduced at a use as a power buffer rate of $3 \mathrm{~kW} / \mathrm{s}$ (though the electrochemistry in fuel cells works on a time scale of milliseconds, the distribution of air and hydrogen is a much slower process limiting the rates of variation of power). The excess power from the fuel cells not needed for traction, together with a part of the kinetic energy, flows into the supercapacitors (negative swing of supercapacitor power in Fig. 4). After passing the curves, the car is again accelerated to the previous speed, mainly using power from the supercapacitors (positive peaks of $30-50 \mathrm{~kW}$ ). As soon as the fuel cells deliver more power than needed to drive the car, their excess power is fed into the supercapacitors to re-establish their previous state of charge ( $90 \%$ of maximum capacity).

\section{A test on the road}

To understand the absolute power levels, an experimental platform termed $H y$. Power was realized by an interdisciplinary team headed by Philipp Dietrich [5-7]. It was based on a Volkswagen BORA chassis and body equipped with an electric engine and the fuel cell power train. From the designed $48 \mathrm{~kW}$ maximum power output of the module consisting of six PEM fuel cell stacks (PEM = Polymer Electrolyte Membrane), about $20 \%$ is needed for auxiliaries. The most energy intensive auxiliary is the compressor providing air at a pressure of about 2 bar ( 1 atmosphere overpressure), to ensure oxygen flow through the $0.3 \mathrm{~mm}$ deep channels of the bipolar stacking plates, which distribute the air across the surface $\left(200 \mathrm{~cm}^{2}\right)$ of the electrodes. At the other electrode, separated from the former by the PEM membrane, hydrogen is supplied from two 350 bar pressure tanks of $26 \mathrm{~L}$ each. In the fuel cell reaction, oxygen and hydrogen are combined to water, and water vapour is the only exhaust of this zero emission vehicle. With a total of $1.1 \mathrm{~kg}$ of hydrogen stored in these tanks, the Hy. Power car would run over about $50-100 \mathrm{~km}$ (simulated for different driving cycles).

As one of the tests for our Hy.Power car, the $2005 \mathrm{~m}$ ( $6580 \mathrm{ft}$.) high Simplon pass connecting Brig ( $680 \mathrm{~m}$ (2230 ft.), Switzerland) with Domodossola (Italy) was chosen. Both with regard to energy and power, the requirements of this test route were about three times more stringent than those of other test runs previously completed by fuel cell vehicles. Not surprisingly Philipp Dietrich and his group experienced extremely hard days and even harder nights to prepare this real world test! During the cold morning of January $16,2002\left(-9^{\circ} \mathrm{C}, 15^{\circ} \mathrm{F}\right)$, the long-expected event took place. The relief on every face was clearly evident when the Hy.Power came round the last curve and arrived on the snowy Simplon pass (see Fig. 5).

\section{References}

[1] R. Kötz and M. Carlen, Principles and applications of electrochemical capacitors, Electrochimica Acta 45, 2483-2498 (2000).

[2] B.B. Mandelbrot, Gaussian Self-Affinity and Fractals, Springer, New York, Berlin, Heidelberg (2002).

[3] H.M. Hastings and G. Sugihara, Fractals - A user's guide for natural sciences, Oxford University Press (1993).

[4] R. Richner, S. Müller, M. Bärtschi, R. Kötz and A. Wokaun, Physically and Chemically Bonded Material for Double-Layer Capacitor Applications, New Materials for Electrochemical Systems 5(3), 297-304 (2002).

[5] P. Dietrich, F. Büchi, A. Tsukada, M. Bärtschi, R. Kötz, G.G. Scherer, P. Rodatz, O. Garcia, M. Ruge, M. Wollenberg, P. Lück, A. Wiartalla, C. Schönfelder, A. Schneuwly and P. Barrade, Hy.Power - A Technology Platform Combining a Fuel Cell System and a Supercapacitor Short Time Energy Storage Device, proc. SATG Konferenz 13.9.02, Will (2002).

[6] P. Dietrich, F. Büchi, A. Tsukada, M. Bärtschi, R. Kötz, G.G. Scherer, P. Rodatz, O. Garcia, M. Ruge, M. Wollenberg, P. Lück, A. Wiartalla, C. Schönfelder, A. Schneuwly and P. Barrade, First Results of the Hy.Power - A Hybrid Fuel Cell Powertrain with a Supercap Energy Storage Device, proc. VDI-Konferenz 24.-25.10.02, Dresden (2002).

[7] P. Rodatz, O. Garcia, L. Guzzella, F. Büchi, M. Bärtschi, A. Tsukada, P. Dietrich, R. Kötz, G.G. Scherer and A. Wokaun, Performance and operational characteristics of a hybrid vehicle powered by fuel cells and supercapacitors, Soc. of Automotive Engineers, SAE paper 2003-010418 (2003). 